\title{
Relating observations of contrail persistence to numerical weather analysis output
}

\author{
D. P. Duda ${ }^{1}$, R. Palikonda ${ }^{2}$, and P. Minnis ${ }^{3}$ \\ ${ }^{1}$ National Institute of Aerospace, Hampton, Virginia, USA \\ ${ }^{2}$ Science Systems and Applications, Inc., Hampton, Virginia, USA \\ ${ }^{3}$ Science Directorate, NASA Langley Research Center, Hampton, Virginia, USA \\ Received: 7 April 2008 - Published in Atmos. Chem. Phys. Discuss.: 21 October 2008 \\ Revised: 23 December 2008 - Accepted: 25 January 2009 - Published: 19 February 2009
}

\begin{abstract}
The potential for using high-resolution meteorological data from two operational numerical weather analyses (NWA) to diagnose and predict persistent contrail formation is evaluated using two independent contrail observation databases. Contrail occurrence statistics derived from surface and satellite observations between April 2004 and June 2005 are matched to the humidity, vertical velocity, wind shear and atmospheric stability derived from analyses from the Rapid Update Cycle (RUC) and the Advanced Regional Prediction System (ARPS) models. The relationships between contrail occurrence and the NWA-derived statistics are analyzed to determine under which atmospheric conditions persistent contrail formation is favored within NWAs. Humidity is the most important factor determining whether contrails are short-lived or persistent, and persistent contrails are more likely to appear when vertical velocities are positive. The model-derived atmospheric stability and wind shear do not appear to have a significant effect on contrail occurrence.
\end{abstract}

\section{Introduction}

Contrail-induced cloud cover has the potential to produce significant regional effects on climate (Minnis et al., 2004). As air traffic increases, the possibility for globally significant impacts also rises. Although these impacts, such as radiative forcing, have been estimated using general circulation models (GCMs) (Marquart and Mayer, 2002; Hansen et al., 2005), their magnitudes remain highly uncertain because

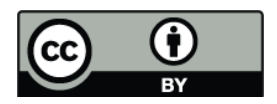

Correspondence to: D. P. Duda (dduda@nianet.org) of the relatively crude ways that contrails are parameterized in those models. One approach to improve contrail representations and to understand and predict contrail climate effects better, is to develop models that can more accurately simulate contrail properties at smaller scales based on ambient atmospheric variables including temperature, relative humidity, and winds and then parameterize those models for inclusion in GCMs. Higher resolution contrail models could also be useful for aiding contrail mitigation efforts, if that need arises. To perform such simulations, it is critical to have meteorological data at high temporal and spatial resolutions over a large domain because air traffic over a given location changes rapidly throughout the day. Only a few operational numerical weather analyses (NWA), including the NOAA/ESRL Rapid Update Cycle (RUC; Benjamin et al., 2004) and the Advanced Regional Prediction System (ARPS; Xue et al., 2003) produced by the University of Oklahoma Center for Analysis and Prediction of Storms, currently provide those variables at the resolutions necessary to diagnose persistent contrail formation over the United States of America.

The basic thermodynamics of contrail formation from jet exhaust was described by Schmidt/Appleman theory (Schumann, 1996), which has been modified more recently to account for the effects of jet aircraft efficiency. This theory describes the temperature and pressure conditions necessary to allow the isobaric mixing of the hot, moist exhaust gases with the cold ambient air to form a contrail. The formation of persistent contrails occurs when the relative humidity with respect to ice (RHI) reaches or exceeds $100 \%$. A review of the historical development of the theory and its experimental verification is given in Schumann (1996). NWAs, however, often underestimate upper tropospheric relative humidity

Published by Copernicus Publications on behalf of the European Geosciences Union. 
GLOBE contrail-reporting schools and cumulative commercial aircraft flight lengths

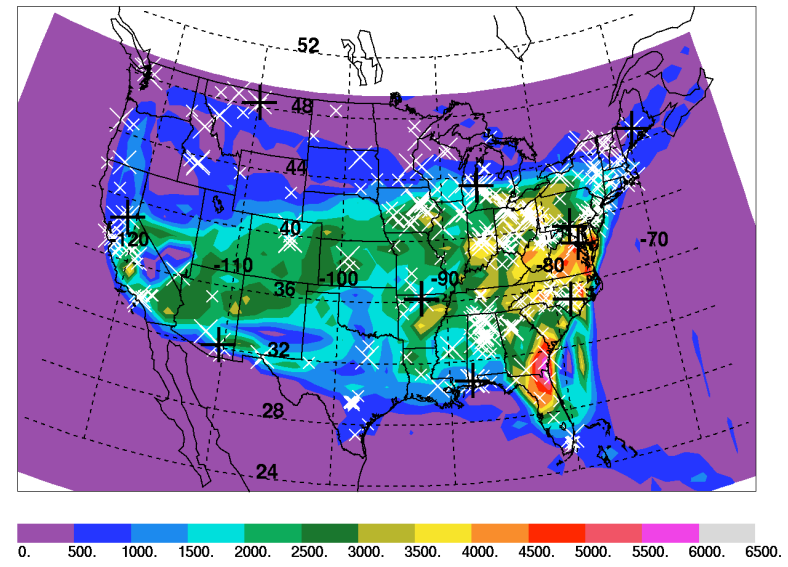

Fig. 1. Distribution of hourly mean cumulative flight lengths (in $\mathrm{km}$ ) for all commercial flights with altitudes between 7 and $15 \mathrm{~km}$ for each $1 \times 1$ degree grid box for the time period between 15:00 and 20:00 UTC during November 2004 (see Garber et al., 2005). The crosses and plus signs indicate all GLOBE locations reporting contrail observations between April 2004 and June 2005. The small white crosses represent locations with 30 or fewer total observations, while the larger white crosses are locations with more than 30 total observations. The black plus signs denote locations with more than 50 observations taken during mostly clear (cloud coverage less than $25 \%$ ) conditions.

(UTH) due to large dry biases in the balloon soundings used to construct the analyses and to internal adjustments made to meet the model's physical constraints (Minnis et al., 2005b). Although this dry bias prevents a straightforward determination of persistent contrail formation via Schmidt/Appleman theory, such a dry bias might be correctable if the numerical weather humidity data can be shown to be consistent with the appearance or non-appearance of contrails. Thus, one outstanding problem that must be addressed before a realistic simulation of contrails can be achieved is to determine how accurately the meteorological data provided by the numerical weather analyses and forecasts diagnose contrail formation conditions.

This paper evaluates the potential for using the RUC and ARPS models to diagnose and predict persistent contrail formation conditions using a variety of datasets. To achieve that goal, we match several months of contrail occurrence statistics derived from satellite and surface observations to the NWA-derived humidity, vertical velocity, wind shear and atmospheric stability. The relationships between contrail occurrence and the NWA-derived statistics are then analyzed to determine under which atmospheric conditions the formation of persistent contrails is favored.

\section{Data and methodology}

Two independent types of contrail observations are used to evaluate the NWA models. Satellite data can reveal contrails above lower level clouds that are missed by surface observers, but are biased, on average, toward lower occurrence rates. Surface observers can provide more reliable contrail observations and detect some of the thinner contrails missed by the satellite.

\subsection{Surface data}

The Global Learning and Observations to Benefit the Environment (GLOBE) program collects observations of cloud occurrence and coverage throughout the contiguous United States (CONUS) from primary and secondary schools across the country. (See www.globe.gov for more information about the GLOBE program.) In May 2003, GLOBE initiated a contrail observation protocol to measure and classify contrail observations. A primary goal of the GLOBE program is to use detailed written protocols to enable students to provide scientifically valuable measurements of environmental parameters (Brooks and Mims, 2001). Over 18500 observations were reported over the region between 1 April 2004 and 27 June 2005. The observations usually include contrail coverage, contrail number, cloud coverage, cloud type and a classification of contrails into three categories, shortlived (SHRT), non-spreading persistent contrails (NSPR), and spreading persistent contrails (SPRD). The contrail categories are defined as follows: short-lived contrails are contrails that dissipate as the aircraft moves across the sky. Persistent contrails are contrails that remain in the sky after the aircraft has flown out of view of the observer. Spreading contrails are defined as persistent contrails wider than the width of a finger held at arm's length. This width corresponds to a contrail that is 2 degrees of arc wide, or at least $350 \mathrm{~m}$ wide (based on a contrail altitude of $10 \mathrm{~km}$ ) (O'Shea, 1991), which is the minimum width expected to be detectable in NOAA's Advanced Very High Resolution Radiometer (AVHRR) imagery. The cloud coverage observations are reported within six categories based on total coverage of non-contrail cloudiness: no clouds ( $0 \%$ coverage), clear (1-9\% coverage), isolated (10-24\% coverage), scattered (25-49\% coverage), broken ( $50-89 \%$ coverage) and overcast ( $90-100 \%$ coverage).

The GLOBE contrail dataset contains observations from 417 schools. The schools are mostly located in highly populated regions with substantial air traffic (Fig. 1). Only 123 of the schools $(29.5 \%)$ reported more than 30 observations during the 15-month time period, but those schools reported 16008 observations, $86.5 \%$ of the total. Nearly all schools reported only one observation/day. Approximately $92 \%$ of all observations were between 14:30 and 20:30 UTC, and nearly $58 \%$ of the total were between 16:30 and 18:30 UTC.

To test the quality of the surface-based observations, the set of 11 schools that had at least 50 observations taken under 
Table 1. Mean of the maximum upper tropospheric relative humidities (in percent) with respect to ice (RHI) collocated with 11 GLOBE surface observation locations under mostly clear skies between April 2004 and June 2005 (number of observations in parentheses). Observations are sorted into four categories based on detection of contrail occurrence by surface and satellite.

\begin{tabular}{llrrrr}
\hline Location & Lat Lon & $\begin{array}{r}\text { A: Surface (Y) } \\
\text { Satellite (Y) }\end{array}$ & $\begin{array}{r}\text { B: Surface (N) } \\
\text { Satellite (Y) }\end{array}$ & $\begin{array}{r}\text { C: Surface (Y) } \\
\text { Satellite (N) }\end{array}$ & $\begin{array}{r}\text { D: Surface(N) } \\
\text { Satellite (N) }\end{array}$ \\
\hline Mobile, AL & $30.70 \mathrm{~N}-88.05 \mathrm{~W}$ & $86.91(8)$ & $84.09(5)$ & $54.10(3)$ & $42.96(48)$ \\
Fayetteville, NC & $35.05 \mathrm{~N}-78.59 \mathrm{~W}$ & $77.47(8)$ & $79.31(5)$ & $81.00(4)$ & $38,94(18)$ \\
Norfork, AR & $36.20 \mathrm{~N}-92.27 \mathrm{~W}$ & $76.35(6)$ & $-(0)$ & $40.65(22)$ & $38.09(25)$ \\
Mountain Home, AR & $36.24 \mathrm{~N}-92.32 \mathrm{~W}$ & $73.43(6)$ & $74.01(2)$ & $38.03(19)$ & $39.94(17)$ \\
Hartland, ME & $44.88 \mathrm{~N}-69.45 \mathrm{~W}$ & $86.58(4)$ & $80.42(1)$ & $63.16(2)$ & $46.01(13)$ \\
Placerville, CA & $38.78 \mathrm{~N}-120.89 \mathrm{~W}$ & $96.20(18)$ & $64.38(3)$ & $31.44(3)$ & $37.80(11)$ \\
Tucson, AZ & $32.17 \mathrm{~N}-110.44 \mathrm{~W}$ & $-(0)$ & $-(0)$ & $39.15(2)$ & $43.64(21)$ \\
Waynesboro, PA & $39.75 \mathrm{~N}-77.57 \mathrm{~W}$ & $96.40(2)$ & $-(0)$ & $25.57(1)$ & $53.32(15)$ \\
Box Elder, MT & $48.29 \mathrm{~N}-109.87 \mathrm{~W}$ & $84.29(1)$ & $74.41(3)$ & $70.58(2)$ & $86.86(10)$ \\
Whitehall, MI & $43.38 \mathrm{~N}-86.32 \mathrm{~W}$ & $92.99(5)$ & $94.52(6)$ & $-(0)$ & $70.59(24)$ \\
Washington, DC & $38.56 \mathrm{~N}-77.01 \mathrm{~W}$ & $94.96(12)$ & $94.92(2)$ & $57.17(12)$ & $54.36(10)$ \\
Total & & $88.19(70)$ & $82.51(27)$ & $46.50(70)$ & $48.26(212)$ \\
\hline
\end{tabular}

mostly clear skies (non-contrail cloud coverage less than $25 \%$ ) were chosen for closer study. Because these schools provided multiple observations throughout the period, we expect that these locations would be more likely to provide high quality contrail observations among the GLOBE participants than locations with few contrail reports. Figure 1 shows the location of the 11 schools as a large black plus (+) sign. All schools with the exception of Box Elder, Montana are located in regions with substantial commercial air traffic during the typical observation period. From these schools, a uniformly random sample of 379 GLOBE contrail observations were compared with observations of contrail occurrence determined by visual inspection of loops of nearly coincident, multi-spectral Geostationary Operational Environmental Satellite (GOES) imagery (Minnis et al., 2008). The comparison between surface and satellite observations is summarized in Table 1, where the observations are sorted into four categories. Category A indicates that both the surface and satellite observations detected contrails, while category D shows the cases where both methods detected no contrails. Category B includes the occasions when contrail occurrence was detected by satellite but not reported by the student observers, and category $\mathrm{C}$ include the observations when the surface observer reported persistent contrails while none were apparent in the satellite imagery. The surface and satellite observations matched nearly $75 \%$ of the time (categories $\mathrm{A}$ and $\mathrm{D}$ ), while $18 \%$ of the observations were in category $\mathrm{C}$, and the remaining $7 \%$ of observations were in category B. As a test of the integrity of the surface and satellite observations, the maximum relative humidity with respect to ice (RHI) within the upper troposphere (between 150 and $400 \mathrm{hPa}$ ) was determined from the ARPS analyses for each observation, and the mean for each observation category is presented in Table 1 . The mean
ARPS RHI for category A observations was $88.2 \%$, while the mean ARPS RHI was only $48.3 \%$ when both observations showed no contrails (category D). It is important to note some differences between surface-based and satellitebased observations. Surface observers often miss contrails forming above lower cloudiness (although by choosing only mostly clear observations, this type of error should be minimal here), misidentify linear cloud features as contrails (or contrails as cloud streaks), or record the observation incorrectly in the contrail report. Cloud cover and the misidentification of cloud streets as contrails also hamper the visual detection of persistent contrails in the GOES satellite imagery loops. Surface observers, however, can detect much narrower and probably optically thinner contrails than those seen in the 4-km resolution GOES satellite imagery. Some of the cases where observers reported contrail coverage not seen in the satellite (category C) may have been due to the observation of non-spreading persistent contrails that could not be detected in the GOES imagery loops. In addition, over $75 \%$ of the category $\mathrm{C}$ reports occurred at only three schools. Due to an ambiguity in the GLOBE contrail reporting form, some schools may have reported short-lived contrails in the contrail coverage fractions, leading to the large number of category $\mathrm{C}$ reports for those locations. The lack of any substantial difference in the ARPS RHI between categories $\mathrm{C}$ and $\mathrm{D}$ at the three schools supports the possibility of this type of error.

\subsection{Satellite data}

Multi-spectral data from the AVHRR onboard the NOAA-16 satellite were used to identify linear contrails (Lee, 1989) following the contrail detection algorithm of Mannstein et al. (1999) as applied by Palikonda et al. (2005). The 
Mannstein et al. (1999) algorithm uses a combination of channel $4(10.8-\mu \mathrm{m})$ and channel $5(12.0-\mu \mathrm{m})$ radiances to identify contrails. Radiance data were collected from 366 mid-afternoon overpasses of the satellite between 16 April 2004 and 27 June 2005 within a $4 \times 6$ degree grid box over eastern Ohio/western Pennsylvania/West Virginia $\left(38^{\circ}-42^{\circ} \mathrm{N} ; 78^{\circ}-84^{\circ} \mathrm{W}\right)$, an area selected because of its heavy commercial air traffic (Garber et al., 2005). During the observation period, contrails were detected within $43.3 \%$ of all available grid boxes, while cirrus was detected within $47.7 \%$ of the available grid boxes. Minnis et al. (2005a) and Palikonda et al. (2005) found that the technique tends to overestimate contrail coverage by $20-40 \%$ mainly because of natural cirrus that appears much like contrails.

\subsection{Meteorological data}

Meteorological data from the high-resolution, hourly RUC20 analyses and ARPS analyses and forecasts provide information on temperature, humidity, pressure, and vertical velocity that is matched with each of the surface and satellite observations. The RUC-20 data have a resolution of $20 \mathrm{~km}$, while the ARPS data were obtained from the $27-\mathrm{km}$ resolution, hourly CONUS domain analyses. The ARPS forecasts are 1-day (16-20 h) , 2-day (40-44 h), and 3-day (64-68 h) forecasts from 16:00 UTC to 20:00 UTC.

To match the surface and meteorological data, data from the RUC analyses closest in time with the contrail observations are bi-linearly interpolated to the location of each observation. An observation is not used if the time difference between the observation and the RUC analysis was greater than $1 \mathrm{~h}$. The level between 400 and $150 \mathrm{hPa}$ with the maximum relative humidity with respect to ice $\left(\mathrm{RHI}_{\max }\right)$ is determined from all levels (spaced at every $25 \mathrm{hPa}$ ) that have a temperature less than or equal to $-40^{\circ} \mathrm{C}$. The temperature constraint was added to eliminate areas where the atmosphere is likely to be too warm to form contrails (Appleman, 1953). Although the observed contrails may have formed at other levels, this level was chosen as the most likely level for contrail formation, and to provide a consistent representation of humidity at typical commercial aircraft flight levels. The probability distribution function of the level of $\mathrm{RHI}_{\max }$ is roughly Gaussian in shape for both the RUC and ARPS analyses with a maximum around $250 \mathrm{hPa}$.

All other meteorological data (including vertical velocity) are selected from this level for comparison with the surface observations of contrail occurrence. The vertical shear of the horizontal wind and the temperature lapse rate for the 25-hPa layer below the level of maximum RHI are also computed. The vertical velocity, the lapse rate (a measure of the atmospheric stability) and the vertical shear are expected to influence the spreading rate of persistent contrails (Jensen et al., 1998).

A similar procedure is used to match the ARPS upper tropospheric data with the surface-based contrail observations.
The RHI is computed from the ARPS fields of potential temperature and specific humidity at the 25 -hPa intervals to determine the level of maximum upper tropospheric humidity.

To compare NWA output with the satellite observations, contrail and cirrus occurrence statistics are derived for each $1 \times 1$ degree grid box within the $4 \times 6$ degree observation domain. Contrail occurrence is determined for each grid box from the automated linear contrail detection algorithm while cirrus occurrence is determined by visual inspection of infrared imagery from each satellite overpass. Meteorological data (including RHI, vertical velocity, wind shear and atmospheric lapse rate) from the RUC and ARPS are then linearly interpolated to the center of each $1 \times 1$ degree box within the domain to correlate the meteorological data with the occurrence of contrails and cirrus. Due to gaps in available meteorological data, and to the satellite viewing angle requirements for the contrail detection method, only 5400 grid box matches were available during the observation period.

\section{Results}

\subsection{Comparison of NWA output with surface observations}

Contrail coverage was reported in $27.0 \%$ of a total of 18504 surface observations. At least one persistent (either spreading or non-spreading) contrail was reported in $17.0 \%$ of the observations. This frequency is slightly higher than Minnis et al. (1993) for the period of highest contrail occurrence, 1993-1994: 15.2\% for unobscured skies. Most of the GLOBE data $(80.1 \%)$ were taken during the non-summer months when contrail coverage is greater. Similar to the US Air Force observers in the Minnis et al. study, the highest contrail frequencies were reported from October through April (18.8\%), while the lowest contrail frequencies were reported from June through September (12.0\%). Most of the persistent contrail observations (71.3\%) were made in partly cloudy skies (either clear skies, or skies with isolated or scattered cloudiness). Table 2 shows the mean value of $\mathrm{RHI}_{\max }$ calculated from the collocated RUC and ARPS analyses for several cloudiness categories.

The increase in $\mathrm{RHI}_{\max }$ with increasing cloud coverage category suggests that the GLOBE cloud and contrail observations were consistent with the numerical weather output, as the largest $\mathrm{RHI}_{\max }$ values occurred when overcast skies were reported. Although most persistent contrails were observed under partly cloudy conditions, the RHIs computed by both numerical weather analyses were higher when persistent contrails formed than when partly cloudy conditions were reported. Table 2 shows that persistent contrails form as expected in high humidity environments, although a comparison between the mean $\mathrm{RHI}_{\max }$ for persistent contrails (the category NSPR+SPRD) with the minimum RHI required by the Schmidt/Appleman criteria (100\%) demonstrates that both models show a dry bias of at least 15 to $38 \%$ when 
Table 2. Mean and standard deviation (in parentheses) of the maximum upper tropospheric relative humidities (in percent) with respect to ice (RHI) from RUC and ARPS analyses and from ARPS 1-day, 2-day and 3-day forecasts collocated with GLOBE surface observations between 1 April 2004 and 27 June 2005. The number of GLOBE observations that were matched to the RUC (R obs.) and ARPS (A obs.) analyses for each cloudiness category are also included. The lines in the table indicating contrails are presented in bold print.

\begin{tabular}{llllllll}
\hline & R obs. & RUC & A obs. & ARPS & 1-day & 2-day & 3-day \\
\hline No cloud & 1783 & $33.78(21.4)$ & 1355 & $51.83(22.2)$ & $47.61(21.8)$ & $57.14(22.4)$ & $59.54(23.9)$ \\
Clear & 2661 & $46.32(25.0)$ & 2178 & $65.73(26.1)$ & $60.18(25.4)$ & $67.63(23.9)$ & $68.20(23.8)$ \\
SHRT only & $\mathbf{1 3 0 2}$ & $\mathbf{4 7 . 0 3 ( 2 4 . 8 )}$ & $\mathbf{1 1 1 6}$ & $\mathbf{6 7 . 0 8}(\mathbf{2 3 . 4})$ & $\mathbf{6 1 . 7 8}(\mathbf{2 4 . 7})$ & $\mathbf{6 9 . 5 0}(\mathbf{2 2 . 1})$ & $\mathbf{7 0 . 4 0}(\mathbf{2 2 . 7})$ \\
Isolated & 2017 & $51.72(26.2)$ & 1655 & $71.32(25.6)$ & $65.25(25.3)$ & $70.62(24.3)$ & $70.91(24.3)$ \\
Scattered & 3168 & $53.87(26.6)$ & 2624 & $72.17(28.2)$ & $66.25(26.5)$ & $70.90(24.6)$ & $70.35(24.6)$ \\
Cirrus & 4472 & $57.63(25.7)$ & 3676 & $78.03(25.2)$ & $71.62(24.2)$ & $74.79(22.4)$ & $73.73(23.0)$ \\
Broken & 3433 & $59.03(27.0)$ & 2902 & $77.76(27.4)$ & $70.61(26.9)$ & $72.99(24.9)$ & $72.20(25.4)$ \\
NSPR only & $\mathbf{8 9 2}$ & $\mathbf{6 2 . 1 8}(\mathbf{2 4 . 6})$ & $\mathbf{7 3 5}$ & $\mathbf{8 2 . 5 7 ( 2 4 . 6 )}$ & $\mathbf{7 5 . 8 8}(\mathbf{2 0 . 9})$ & $\mathbf{7 7 . 8 0}(\mathbf{2 0 . 4})$ & $\mathbf{7 5 . 4 5}(\mathbf{2 3 . 1})$ \\
NSPR + SPRD & $\mathbf{2 2 9 4}$ & $\mathbf{6 2 . 2 7 ( 2 4 . 9 )}$ & $\mathbf{1 8 9 5}$ & $\mathbf{8 4 . 6 6 ( 2 2 . 4 )}$ & $\mathbf{7 7 . 5 8}(\mathbf{2 0 . 8})$ & $\mathbf{7 9 . 1 3 ( 2 0 . 2 )}$ & $\mathbf{7 7 . 1 9}(\mathbf{2 2 . 0})$ \\
SPRD only & $\mathbf{1 0 0 1}$ & $\mathbf{6 2 . 0 8 ( 2 5 . 4 )}$ & $\mathbf{8 3 4}$ & $\mathbf{8 5 . 5 9}(\mathbf{2 2 . 7})$ & $\mathbf{7 7 . 5 4 ( 2 1 . 1 )}$ & $\mathbf{7 9 . 3 5}(\mathbf{2 0 . 7})$ & $\mathbf{7 7 . 7 9}(\mathbf{2 1 . 3})$ \\
Overcast & 5060 & $\mathbf{6 5 . 8 5 ( 2 7 . 5 )}$ & 4247 & $85.63(27.5)$ & $74.43(26.8)$ & $73.94(26.4)$ & $71.48(27.1)$ \\
\hline
\end{tabular}

compared to contrail formation theory. The contrail observations with the highest average $\mathrm{RHI}_{\max }$ conditions occurred when persistent spreading contrails were present. The dry bias is expected as both models limit upper tropospheric moisture. Even though ice supersaturation is common in the upper troposphere and RHI has been measured to be as high as $150 \%$ (e.g., Miloshevich et al., 2001), both the RUC and the ARPS analyses contain at most only slight ice supersaturations which likely appear incidentally as the result of numerical issues. The RUC-20 analyses do not allow RHI to exceed $100 \%$ by more than a few percent at pressures below $300 \mathrm{hPa}$. The RUC-20 mean value of RHI for overcast clouds is $\sim 15 \%$ less than that found by Minnis et al. (2005b) when comparing RUC-2 RHI with clouds having temperatures below $-40^{\circ} \mathrm{C}$. This difference reflects the change in upper tropospheric humidity processing scheme between RUC-2 and RUC-20 to limit RHI artificially in the RUC-20. The ARPS analyses allow some ice supersaturation, although RHI values rarely exceed $112 \%$. No ice supersaturation occurs in the ARPS forecasts; the maximum RHI is only 100 percent. This difference between the analyses and forecasts could account for the marked drop in the RHI values in the forecasts shown in Table 2 .

Table 3 shows the mean values of vertical velocity (vv), vertical wind shear (vs), and atmospheric lapse rate (lr) from the RUC and ARPS collocated with the surface contrail observations from GLOBE. Although most persistent contrails were reported under partly cloudy conditions, the RUC and ARPS vertical velocities were larger than the mean vertical velocities reported under such cloudiness conditions. When persistent contrails were present, the vertical shear of the horizontal wind was similar to the shear analyzed under typical partly cloudy conditions. The temperature lapse rate at the level of maximum RHI indicates the stability of the atmosphere where contrails form, and helps determine the
Table 3. Mean vertical velocity in $\mathrm{cm} \mathrm{s}^{-1}$ (vv), mean vertical shear of the horizontal wind in $\mathrm{m} \mathrm{s}^{-1} \mathrm{~km}^{-1}$ (vs), and mean lapse rate in $\mathrm{K} \mathrm{km}^{-1}$ (lr) computed from RUC and ARPS analyses collocated with GLOBE surface observations between April 1, 2004 and June 27,2005 . The lines in the table indicating contrails are presented in bold print.

\begin{tabular}{lcclllll}
\hline & \multicolumn{2}{c}{$\mathrm{vv}\left(\mathrm{cm} \mathrm{s}^{-1}\right)$} & \multicolumn{2}{c}{$\mathrm{vs}\left(\mathrm{m} \mathrm{s}^{-1} \mathrm{~km}^{-1}\right)$} & \multicolumn{2}{c}{$\operatorname{lr}\left(\mathrm{K} \mathrm{km}^{-1}\right)$} \\
& RUC & ARPS & RUC & ARPS & RUC & ARPS \\
\hline No cloud & -1.16 & -0.66 & 3.94 & 3.42 & -6.77 & -6.00 \\
Clear & -0.71 & -0.53 & 4.10 & 3.66 & -7.01 & -6.43 \\
SHRT only & $\mathbf{- 0 . 5 6}$ & $\mathbf{- 0 . 5 7}$ & $\mathbf{3 . 8 4}$ & $\mathbf{3 . 5 5}$ & $-\mathbf{7 . 1 1}$ & $-\mathbf{6 . 5 6}$ \\
Isolated & -0.18 & -0.18 & 4.11 & 3.57 & -7.12 & -6.82 \\
Scattered & +0.04 & -0.31 & 4.11 & 3.60 & -7.07 & -6.85 \\
Cirrus & +0.11 & -0.15 & 4.12 & 3.73 & -7.24 & -7.02 \\
Broken & +0.81 & +0.20 & 4.22 & 3.79 & -7.14 & -7.06 \\
NSPR only & $\mathbf{+ 0 . 5 0}$ & $\mathbf{+ 0 . 0 5}$ & $\mathbf{4 . 2 2}$ & $\mathbf{3 . 7 0}$ & $-\mathbf{7 . 3 3}$ & $\mathbf{- 7 . 0 7}$ \\
NSPR+ SPRD & $\mathbf{+ 0 . 3 7}$ & $\mathbf{+ 0 . 1 0}$ & $\mathbf{4 . 1 8}$ & $\mathbf{3 . 6 6}$ & $-\mathbf{7 . 3 5}$ & $\mathbf{- 7 . 1 8}$ \\
SPRD only & $\mathbf{+ 0 . 3 1}$ & $\mathbf{+ 0 . 1 9}$ & $\mathbf{4 . 1 4}$ & $\mathbf{3 . 6 6}$ & $-\mathbf{7 . 3 4}$ & $\mathbf{- 7 . 2 8}$ \\
Overcast & $\mathbf{+} .05$ & $\mathbf{+ 1 . 0 4}$ & 4.57 & 4.22 & -7.24 & -7.19 \\
\hline
\end{tabular}

depth of persistent contrails. Contrails are expected to be thicker vertically as the magnitude of the lapse rate increases (i.e., the atmosphere becomes less stable). Although Table 3 shows that the largest mean lapse rates occur in both the RUC and ARPS analyses when spreading persistent contrails are reported, the differences in the lapse rate are not large enough to be statistically significant.

\subsection{Comparison of NWA output with satellite observations}

Figure 2 presents normalized probability density histograms of RHI computed using the $1 \times 1$ degree grid boxes from the satellite overpasses. In Fig. 2a, RHI values from the RUC and ARPS models are separated into grid boxes containing 


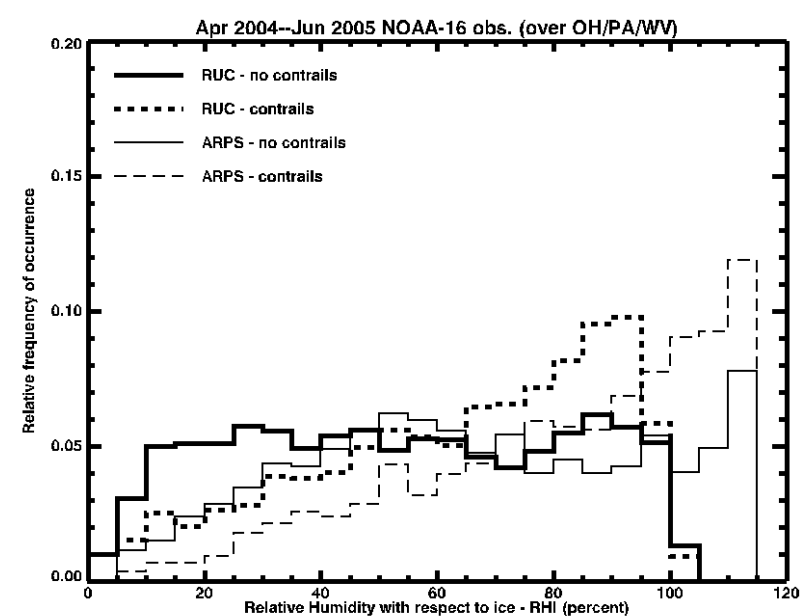

Fig. 2a. Normalized probability distributions of relative humidity with respect to ice (RHI) computed from RUC and ARPS analyses collocated with NOAA-16 observations of the occurrence or non-occurrence of persistent contrails between 1 April 2004 and 27 June 2005.

Table 4. Optimum RHI-based ARPS and RUC forecast skill statistics determined from NOAA-16 measurements of cirrus and contrail occurrence for $\mathrm{OH} / \mathrm{PA} / \mathrm{WV}$ region from 366 afternoon overpasses from 16 April 2004 to 27 June 2005.

\begin{tabular}{lllll}
\hline Cirrus & Optimum RHI & Hit Rate & Bias Ratio & HSS \\
\hline ARPS & 78 & 0.779 & 0.967 & 0.556 \\
RUC & 62 & 0.756 & 1.004 & 0.512 \\
ARPS 1-day forecast & 76 & 0.732 & 0.960 & 0.461 \\
ARPS 2-day forecast & 76 & 0.632 & 1.092 & 0.265 \\
ARPS 3-day forecast & 80 & 0.552 & 0.933 & 0.097 \\
Contrail & & & & \\
ARPS & 74 & 0.614 & 1.159 & 0.228 \\
RUC & 62 & 0.589 & 1.106 & 0.173 \\
ARPS 1-day forecast & 70 & 0.613 & 1.146 & 0.227 \\
ARPS 2-day forecast & 77 & 0.572 & 1.123 & 0.143 \\
ARPS 3-day forecast & 80 & 0.545 & 0.985 & 0.075 \\
\hline
\end{tabular}

contrails (dashed lines) and boxes with no contrails (solid lines). The contrail distributions are skewed toward higher RHI values, but the "no contrail" distributions are relatively uniform. The distributions in Fig. 2b, which are separated by the presence or absence of cirrus, show a clear distinction in the humidity between the cirrus and non-cirrus grid boxes. Figure $2 b$ suggests that the NWAs may be better at predicting cirrus than contrail occurrence. The demarcation seen in Fig. $2 b$ suggests that for the ARPS analyses, a simple threshold near 75\% RHI would accurately predict cirrus occurrences while for the RUC analyses the best threshold would be between 60 and $65 \%$.

To assess the skill level of such an RHI-based forecast, three measures of forecasting skill were computed. The skill scores were constructed using a simple 2-by-2 forecast matrix with the following outcomes: $a$ is the number of cases

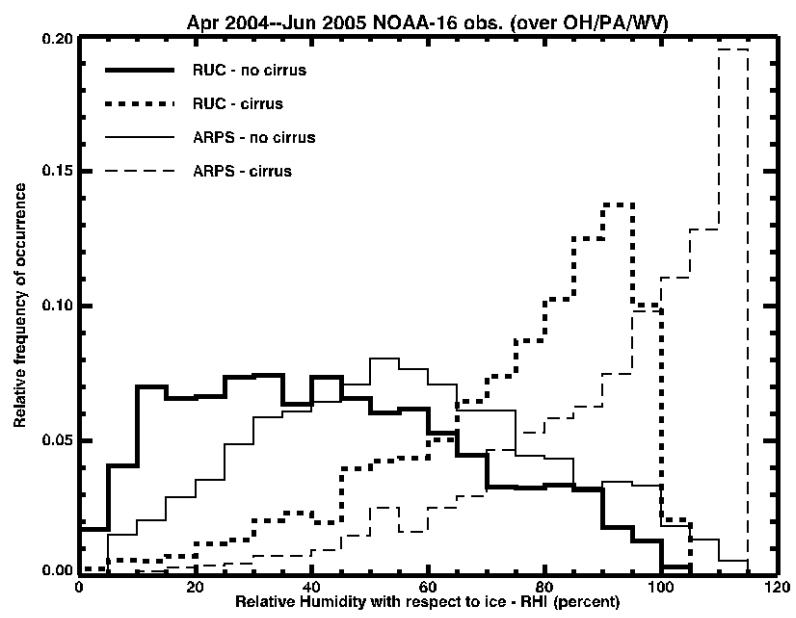

Fig. 2b. Normalized probability distributions of relative humidity with respect to ice (RHI) computed from RUC and ARPS analyses collocated with NOAA-16 observations of the occurrence or nonoccurrence of cirrus between 1 April 2004 and 27 June 2005.

where the RHI is at or above the threshold and cloud is observed in the grid box (hits); $b$ is the number of cases where RHI is at or above the threshold but no cloud is observed (false alarms); $c$ is the number of cases where RHI is below the threshold but a cloud is observed (misses); and $d$ is the number of cases where RHI is below the threshold and no cloud is observed (correct rejections). The three skill measures are:

Hit rate. The hit rate is $(a+d) /(a+b+c+d)$, and represents the percentage of forecasts in which the method correctly predicted the observed event.

Bias ratio. The bias ratio is computed from $(a+b) /(a+c)$, and measures the tendency of a forecast method to overor under-forecast the occurrence of contrails or cirrus. A perfectly unbiased method would have a bias ratio of 1.00 , while values below unity indicate that the cirrus/contrail occurrence is under-forecasted, and values above unity indicate that cirrus/contrail occurrence is over-forecasted.

Heidke Skill Score (HSS). The HSS is calculated as HSS $=2(a d-b c) /[(a+c)(c+d)-(a+b)(b+d)]$ (see Wilks, 1995). This measure of forecasting skill compares the hit rate of the forecast method with the hit rate achieved with a random forecast. Perfect forecasts have an HSS of one, forecasts equal in skill to the random forecast have an HSS of zero, while a negative HSS indicates that the forecasts are less skillful than random forecasts.

Table 4 shows the hit rate, bias ratio and HSS computed for the best-case RHI threshold chosen for each NWP analysis/forecast for both cirrus and contrail occurrence. The optimal RHI for each case was determined by finding the particular RHI threshold that maximizes HSS. The HSS-optimized thresholds also tended to have the highest hit rates, and the bias ratios usually were close to 1.00 . 
To test the significance of the HSS in the contrail cases (compared to a random forecast), the variance of HSS within 1000 series of random contrail predictions were compared to the HSS computed for the contrail cases. For each series, random contrail predictions were determined for each satellite grid box using uniformly distributed random numbers and the mean contrail occurrence within the grid boxes (approximately 0.46). The HSS of the random forecasts were computed for each series, and the distribution of HSS for the 1000 runs were plotted to determine the variance of the HSS. The variance of HSS in the random forecasts is a function of the total number of contrail forecasts (i.e., grid boxes) within a series. For the RUC and ARPS analyses (5401 grid boxes), the HSS was no more than +0.050 and no less than -0.043 . The ARPS 3-day forecasts had the smallest number of available grid boxes (2385), so that the range in HSS for the random 3-day forecasts ranged from +0.067 to -0.053 . We expect that the HSS in all of the contrail cases (except perhaps the 3-day forecasts, which had an HSS equal to 0.075) are beyond at least three times the standard deviation of the HSS in the random forecasts, and thus are statistically significant.

As might be expected based on an inspection of Fig. 2, the cirrus occurrence forecasts were much better than the contrail occurrence forecasts for both analyses. Because cirrus and contrails can only form in supersaturated regions, the optimal RHI should be at least $100 \%$. Thus, both the RUC and the ARPS have a dry bias. Some dry bias is expected because the model analyses produce a grid average relative humidity such that the model would have an RHI of $100 \%$ only when the entire grid box were covered in clouds, which usually does not occur when persistent contrails are observed from the surface. The difference in the skill scores between the RUC and ARPS analyses is not as large as the difference in the cirrus skill scores compared to the contrail skill scores. The higher cirrus skill scores suggest that the model analyses do a better job representing the areas of UTH associated with cirrus than regions where persistent contrails appear. This may be related to the difficulty of the RUC and ARPS to assimilate contrail observations in their analyses.

\section{Discussion and conclusions}

The results in Table 2 confirm that relative humidity is the most important factor determining whether contrails are short-lived or persistent. Although most surface observations of contrails occur in clear or partly cloudy skies, the UTH observed when persistent contrails form is typically much higher than the average humidities observed under partly cloudy conditions, or when only short-lived contrails are reported. In contrast, the RHI values reported when spreading persistent contrails are observed are nearly the same as the RHI when non-spreading persistent contrails are reported.

Vertical velocity also appears to influence where persistent contrails may form. Persistent contrails are more likely to ap- pear when the vertical velocities are positive than when negative. Although both vertical shear and atmospheric lapse rate probably influence contrail spreading, these two variables appear to be less important in determining whether contrails will spread than the other factors. It is likely that sufficient UTH and positive vertical velocities help to make contrails deep enough and long-lived so that the wind shear can spread the clouds. Another result of this study is that the upper tropospheric RHI in the RUC/ARPS analyses correlates well with satellite observations of cirrus (probably due to the assimilation of cirrus coverage in the models), but the prognosis of contrails from relative humidity analyses is complicated by the occasional lack of contrail formation when UTH is high. This may be the result of cirrus clouds competing with contrails for atmospheric moisture and obscuring the detection of any contrails that formed. It might also be due to errors in the UTH diagnosed by the models, or simply to the lack of jet air traffic in the region. Other factors that can affect the determination of contrails or cirrus from NWAs are input data such as satellite radiances or cloudtop heights. Inclusion of those parameters into the numerical weather assimilation can, in many instances, force the model to increase or decrease the UTH to match the observations resulting in the relatively good diagnoses of cirrusrelated parameters seen here. However, such data are available only in an analysis model and would only contribute to the diagnoses in a forecast model indirectly by improving the forecast. A comparison of the forecast skill statistics in Table 4 shows that the simple RHI threshold model loses much forecast skill in predicting cirrus occurrence after $24 \mathrm{~h}$.

Because the intent of this paper is to demonstrate the overall applicability of NWA data to predict contrail occurrence, no consideration is given here to the spatial or seasonal variations in these atmospheric variables. More insight may be gained in future studies by looking at spatial or seasonal differences in the contrail occurrence data.

Although the meteorological data within the RUC and ARPS analyses are qualitatively consistent with contrail formation theory, direct quantitative agreement with Schmidt/Appleman theory is not currently possible due to the artificial upper limits placed on UTH in both analyses. This arbitrary cutoff of supersaturation removes information about the humidity field that prevents a straightforward application of Schmidt/Appleman theory, which requires accurate temperature and relative humidity data. The results of Table 4 demonstrate that forecast models based on RUC/ARPS humidity alone have limited skill in diagnosing contrail occurrence. Despite these results, the humidity data do show some consistency with persistent contrail occurrence, and it is likely that contrail forecasts could be improved by using logistic regression (Jackson et al., 2001; Travis et al., 1997) or other statistical methods that can include several meteorological parameters in the prognosis. We plan such a multivariable analysis in an ongoing study. In addition, Burkhardt et al. (2008) used assumptions about the subgrid variability in 
relative humidity in a general circulation model (ECHAM4) to diagnose contrail coverage without explicitly representing ice supersaturation in the model. Such a method could be useful in determining contrail occurrence and coverage within the RUC and ARPS analyses. However, we caution that because upper tropospheric humidity is the main factor determining contrail formation, contrail forecasts will remain somewhat limited in accuracy until accurate assimilation of ice supersaturation is incorporated into numerical weather analyses and models. Some current numerical weather prediction models such as the ECMWF IFS (Integrated Forecasting System) model now include supersaturation over the ice phase explicitly (Thompkins et al., 2007), and it is encouraging that the latest versions of both the RUC and the ARPS at the time of this writing are now producing significantly greater levels of ice supersaturation than were available for comparisons with the observations used in this study.

Acknowledgements. This material is based upon work supported by the NASA Earth Science Enterprise Radiation Sciences Division, the NASA Modeling, Analysis, and Prediction (MAP) Program, NASA contracts NAG1-02044 and NCCI-02043 NIA-2579, and by the National Science Foundation under Grant No. 0222623.

Edited by: R. Vautard

\section{References}

Appleman, H.: The formation of exhaust condensation trails by jet aircraft, Bull. Amer. Meteor. Soc., 34, 14-20, 1953.

Benjamin, S. G., Dévényi, D., Weygandt, S. S., Brundage, K. J., Brown, J. M., Grell, G. A., Kim, D., Schwartz, B. E., Smirnova, T. G., Smith, T. L., and Manikin, G. S.: An hourly assimilationforecast cycle: The RUC, Mon. Weather Rev., 132, 495-518, 2004.

Brooks, D. R. and Mims III, F. M.: Development of an inexpensive handheld LED-based Sun photometer for the GLOBE program, J. Geophys. Res., 106(D5), 4733-4740, 2001.

Burkhardt, U., Kärcher, B., Ponater, M., Gierens, K., and Gettelman, A.: Contrail cirrus supporting areas in model and observations, Geophys. Res. Lett., 35, L16808, doi:10.1029/2008GL034056, 2008.

Garber, D., Minnis, P., and Costulis, P. K.: A commercial flight track database for upper tropospheric aircraft emission studies over the USA and southern Canada, Meteorol. Z., 14, 445-452, 2005.

Hansen, J., Sato, M., Ruedy, R., Nazarenko, L., Lacis, A., Schmidt, G. A., Russell, G., Aleinov, I., Bauer, M., Bauer, S., Bell, N., Cairns, B., Canuto, V., Chandler, M., Cheng, Y., Del Genio, A., Faluvegi, G., Fleming, G., Friend, A., Hall, T., Jackman, C., Kelley, M., Kiang, N., Koch, D., Lean, J., Lerner, J., Lo, K., Menon, S., Miller, R., Minnis, P., Novakov, T., Oinas, V., Perlwitz, Ja., Perlwitz, Ju., Rind, D., Romanou, A., Shindell, D., Stone, P., Sun, S., Tausnev, N., Thresher, D., Wielicki, B., Wong, T., Yao, M., and Zhang, S.: Efficacy of climate forcings, J. Geophys. Res., 110, D18104, doi:10.1029/2005JD005776, 2005.

Jackson, A., Newton, B., Hahn, D., and Bussey, A.: Statistical contrail forecasting, J. Appl. Meteor., 40, 269-279, 2001.
Jensen, E. J., Ackerman, A. S., Stevens, D. E., Toon, O. B., and Minnis, P.: Spreading and growth of contrails in a sheared environment, J. Geophys. Res., 103, 31557-31567, 1998.

Lee, T. F.: Jet contrail identification using the AVHRR infrared split window, J. Appl. Meteorol., 28, 99-3-995, 1989.

Mannstein, H., Meyer, R., and Wendling, P.: Operational detection of contrails from NOAA-AVHRR data, Intl. J. Remote Sens., 20, 1641-1660, 1999.

Marquart, S. and Mayer, B.: Towards a reliable GCM estimation of contrail radiative forcing, Geophys. Res. Lett., 29(8), 1179, doi:10.1029/2001GL014075, 2002.

Miloshevich, L. M., Vömel, H., Paukkunen, A., Heymsfield, A. J., and Oltmans, S. J.: Characterization and correction of relative humidity measurements from Vaisala RS80-A radiosondes at cold temperatures, J. Atmos. Oceanic Technol., 18, 135-156, 2001.

Minnis, P., Ayers, J. K., Nordeen, M. L., and Weaver, S. P.: Contrail frequency over the United States from surface observations. J. Climate, 16, 3447-3462, 2003.

Minnis, P., Ayers, J. K., Palikonda, R., and Phan, D.: Contrails, cirrus trends, and climate, J. Climate, 17, 1671-1683, 2004.

Minnis, P., Palikonda, R., Walter, B. J., Ayers, J. K., and Mannstein, H.: Contrail properties over the eastern North Pacific from AVHRR data, Meteorol. Z., 14, 515-523, 2005a.

Minnis, P., Yi, Y., Huang, J., and Ayers, J. K.: Relationships between radiosonde and RUC-2 meteorological conditions and cloud occurrence determined from ARM data, J. Geophys. Res., 110, D23204, doi:10.1029/2005JD006005, 2005 b.

Minnis, P., Young, D., Nguyen, L., Smith, W., Garber, D., Doelling, D., Duda, D., Chang, F.-L., Arduini, R., Avey, L., Ayers, J. K., Brown, R., Chakrapani, V., Spangenberg, D., Gibson, S., Houser, S., Huang, J., Chee, T., Khaiyer, M., Miller, W., Nordeen, M., Palikonda, R., Chen, Y., Sun-Mack, S., Trepte, Q., Yi, H., Yost, C., and Heck, P.: Satellite imagery and cloud products page, http: //www-angler.larc.nasa.gov/satimage/products.html, last access: 17 October, 2008.

O'Shea, R. P.: Thumb's rule tested: visual angle of thumb's width is about 2 deg, Perception, 20(3), 415-418, doi:10.1068/p200415, 1991.

Palikonda, R., Minnis, P., Duda, D. P., and Mannstein, H.: Contrail coverage derived from 2001 AVHRR data over the continental United States of America and surrounding areas, Meteorol. Z., 14, 525-536, 2005.

Schumann, U.: On conditions for contrail formation from aircraft exhausts. Meteor. Z., 5, 4-23, 1996.

Tompkins, A. M., Gierens, K., and Rädel, G.: Ice supersaturation in the ECMWF Integrated Forecast System. Q. J. R. Meteorol. Soc., 133, 53-63, 2007.

Travis, D. J., Carleton, A. M., and Changnon, S. A.: An empirical model to predict widespread occurrences of contrails, J. Appl. Meteor., 36, 1211-1220, 1997.

Wilks, D. S.: Statistical Methods in the Atmospheric Sciences, International Geophysics Series, vol. 59, Academic Press, San Diego, 467 pp., 1995.

Xue, M., Wang, D.-H., Gao, J. -D., Brewster, K., and Droegemeier, K. K.: The Advanced Regional Prediction System (ARPS), storm-scale numerical weather prediction and data assimilation, Meteor. Atmos. Phys., 82, 139-170, 2003. 